\title{
Research Article \\ Effects of the Splitter Blade on the Performance of a Pump-Turbine in Pump Mode
}

\author{
Guidong Li $(\mathbb{D}$, Yang Wang, Puyu Cao, Jinfeng Zhang, and Jieyun Mao \\ National Research Center of Pumps, Jiangsu University, Zhenjiang 212013, Jiangsu, China \\ Correspondence should be addressed to Guidong Li; liguidong1201@163.com
}

Received 12 June 2018; Revised 4 August 2018; Accepted 27 September 2018; Published 11 October 2018

Academic Editor: Zhengbiao Peng

Copyright (C) 2018 Guidong Li et al. This is an open access article distributed under the Creative Commons Attribution License, which permits unrestricted use, distribution, and reproduction in any medium, provided the original work is properly cited.

\begin{abstract}
The pumped hydro energy storage is the most effective way to store large-scale electricity and has been widely used in the world. As the key equipment in the pumped hydro energy storage, it is significant and urgent to improve the performance and operation stability of the pump-turbine. In this study, the effect of runners with and without splitter blade on the performances and inner flow characteristics of a pump-turbine in pump mode was analyzed by the method of numerical calculation. The results suggest that larger tangential velocity at runner outlet and higher pressure at the trailing edge of pressure side in splitter blade runner scheme contribute to higher head. The area of backflow at runner outlet, the highest values of entropy generation rate, and vorticity distribution in splitter blade runner scheme are well smaller than those in prototype runner without splitter blade, which is conducive to improving model performance.
\end{abstract}

\section{Introduction}

Clean energies, such as wind energy, solar energy, nuclear energy, and hydropower, are starting to play a significant role in the process of social development due to the progressive exhaustion of fossil fuels (coal and petroleum, etc.) and the severe environment pollution. However, a new issue has appeared: how to feed these energies to the current electricity system according to the changeable electricity consumption. The pumped hydro energy storage is a good solution to this issue. As a result, it is widely used to keep a balance between the electricity production and consumption and improve the electricity use efficiency and the stability of power grid. There are two reversible storage and generation working modes in the pumped storage power plant as shown in Figure 1. It is a special kind of hydropower with very high efficiency that can convert the electricity to the potential energy of water at low power grid loads (pump mode) and generate electricity with the stored water at high power grid loads (turbine mode) $[1,2]$. As the most promising and effective way to store electricity, the pumped storage power plant has been widely used in Japan, America, Europe, China, and so on [3-5].
The key equipment of pumped storage power plant is the pump-turbine, which can be used as a pump or turbine at different operation conditions and switch between two working modes rapidly. Therefore, some high requirements on the design stage of the pump-turbine are proposed to improve the performance and cavitation ability, keeping stable during start-up and modes switches [6]. Some researchers have worked on the unstable characteristics, rotor-stator interaction, flow patterns, cavitation, and so on of the reversible pump-turbine, contributing to the rapid development and application of pump-turbines. Yan et al. [7] investigated the effect of water compressibility on the rotor-stator interaction by using simulation and experiment. The results showed that the compressible calculation had more accurate pressure fluctuations prediction in vaneless region but higher pressure values in penstock and spiral case. Olimstad et al. [8] studied the effect of different runner leading edge (curvature radius and inlet blade angle) on the characteristics of pump-turbine in turbine mode. The results showed that larger radius and smaller inlet blade angle provided less steep characteristics; however the latter also exacerbated the unstable curves in both turbine and pump modes. Yin et 
al. [9] found that broadening the meridional passage in the runner of pump-turbine could suppress the form of unstable curve. Yang et al. [10] adopted the inverse design method combining with computational fluid dynamics to design the blade profile according to the blade loading distributions and a design-of-experiment method to decide the test points, which was validated by experiment to be effective. Ješe et al. [11] studied the hump-shaped curve and rotating stall in a high head reversible pump-turbine at different guide vane openings. The results indicated that the hump had a great relationship with the rotating stall and at larger opening the hump was lighter. Zhang et al. [12] compared the dynamic and steady characteristics in a pump-turbine by coupling with the corresponding water delivery system during runaway. The results presented the fact that the dynamic method gave more similar key transient parameters and unstable behaviors which influenced looping trajectories.

The splitter blade runner, a special structure runner with long and short blades placed alternately in the circumference direction, was proposed in centrifugal pumps $[13,14]$ and compressors $[15,16]$. It has advantages of improving hydraulic performance and reducing the blade load, the appearance of secondary flow, pressure fluctuation, vibration, and so on. The first pump-turbine with a splitter blade runner was successfully applied in the real power plant in 2003 manufactured by Toshiba Corporation [17]; however there were a few studies on the pump-turbine. Tezuka [18] proposed a design method of splitter blade with the blade inlet equal and outlet unequal pitch arrangement. By using this method, two different specific-speed runners in Azumi and Karmagawa pumped storage power plants were designed, installed, and verified to have high reliability and better hydraulic performance. Meng et al. [19] studied the influence of splitter blade length on the pump-turbine efficiency and pressure fluctuation. The results suggested that the pressure fluctuation was lower with relatively high efficiency when the length ratio of splitter blade to long blade equaled 0.85 .

With the development of computational fluid dynamics, numerical calculation technique as a significant approach of prediction and analysis has been reported in the fluid machinery [20, 21]. Therefore, after summarizing previous studies, this paper conducts a more detailed mechanism study of the inner flow characteristics of the pump-turbine in pump mode and takes into account the effect of splitter blade runner scheme on the performance. The numerical simulations are performed by ANSYS-CFX code. In addition, the simulation calculations are contrasted with experimental results at different flow rates. The velocity contours, pressure distribution, entropy generation rate, and vorticity distribution are analyzed to illustrate the differences of two schemes. Finally, this paper will provide some references on splitter blade design in pump-turbines to improve the performance.

\section{Numerical Simulation Method}

2.1. Geometry Model. A reduced scale of a reversible pumpturbine, with nominal flow rate of $Q_{d}=410 \mathrm{~kg} / \mathrm{s}$, nominal head of $H_{\mathrm{d}}=50 \mathrm{~m}$, and a rotating speed of $1,300 \mathrm{r} / \mathrm{min}$,
TABLE 1: Mesh information.

\begin{tabular}{lcc}
\hline Domain & $\begin{array}{c}\text { Elements } \\
\text { number } \\
\text { (thousand) }\end{array}$ & $\begin{array}{c}\text { Quality } \\
\text { (determinant 2x2x2) }\end{array}$ \\
\hline Runner (prototype) & 2166.7 & 0.56 \\
Runner (splitter blade scheme) & 2161.7 & 0.68 \\
Draft tube & 1674.8 & 0.72 \\
Guide vanes & 1003.6 & 0.89 \\
Stay vanes & 1478.3 & 0.56 \\
Spiral casing & 1124.5 & 0.56 \\
\hline
\end{tabular}

is studied in this paper. The three-dimensional geometric model of the pump-turbine is showed in Figure 2, composed of five components: spiral casing, stay vanes, guide vanes, runner, and draft tube, corresponding to the real machine. It has 20 stay vanes and 20 guide vanes. Figure 3 shows the schemes of two runners without and with splitter blade. The prototype runner has nine main blades whereas the splitter blade runner has six main blades and six splitter blades, respectively. The splitter blade is distributed at the middle of passage and the leading edge is located at 0.45 -time radius of runner.

2.2. Mesh Generation. The pump-turbine computational domain was composed of five parts as the geometry model. The mesh was generated by means of ANSYS-ICEM code, as shown in Figure 4. As the mesh quality has a significant relationship with the calculation result, the hexahedral structured grid was adopted to obtain the results with less element number, short computing time, and small truncation error. The number and location of nodes on each pair of interfaces were refined for overcoming the interface influence on the flow fields and improving calculation accuracy. Furthermore, the mesh was refined near solid wall surface, such as blade and vane surfaces, where it is easy to capture the pressure gradients and flow separation phenomenon. Since the calculation result is sensitive to the grid number, several different sets of grids with increasing numbers were generated to perform the mesh independence analysis at two flow rates for the prototype pump-turbine, as shown in Figure 5. The heads at two different flow rates exhibit larger deviations from the test results when the total mesh elements are less than 6121.3 thousand. After this mesh number, the head gets closer to the test and behaves almost flat with mesh elements increase. As a result, the grid number for the whole computation domain was chosen to approximate 7447.9 thousand. The detailed mesh information of each component for two schemes is listed in Table 1.

2.3. Numerical Simulation Setup. The numerical simulation was carried out with commercial ANSYS-CFX code by solving the Reynolds-averaged Navier-Stokes (RANS) equations based on the finite volume method. Shear stress transport (SST) model $[21,22]$ was complemented to 


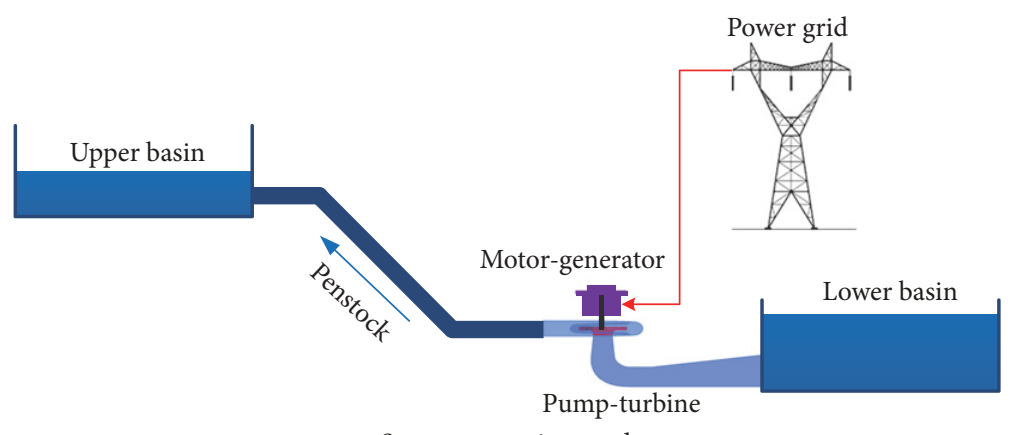

Storage-pumping mode



FIGURE 1: Electricity storage and generation in pumped storage power plant.

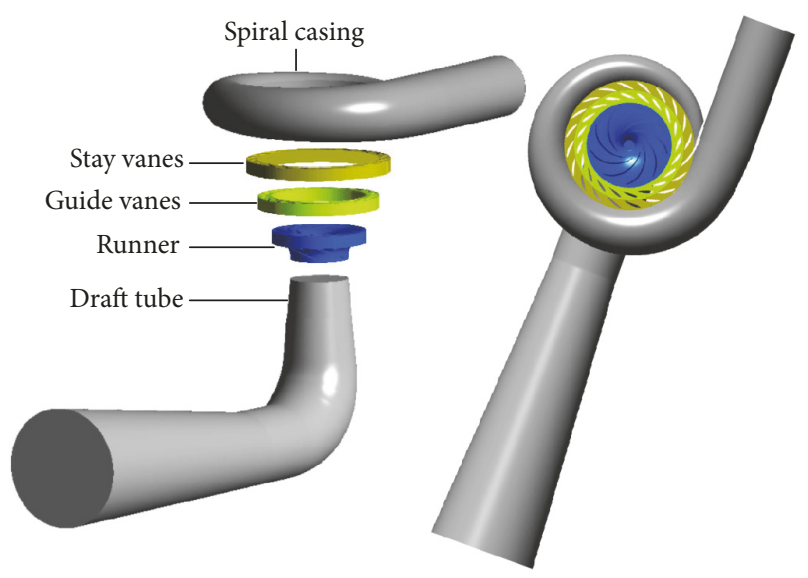

FIgURE 2: The three-dimensional geometric model of the pumpturbine.

simulate turbulence flow in the pump-turbine and automatic wall function was chosen for the near wall treatment. The discretization methods for time and space were selected as second-order backward Euler and high resolution, respectively. The rotating reference frame was applied to the runner while the stationary reference frame was installed at other parts. In addition, total pressure was imposed as boundary condition at the draft tube inlet. The mass flow with stochastic fluctuation of the velocity was installed at the spiral casing outlet, in sympathy with test condition. The calculation was considered to be convergence with the root mean square (RMS) residual less than $10^{-5}$.

\section{Experimental Validation}

In this study, the performance experiments of pump-turbine model were carried out at one guide vane opening $(25 \mathrm{~mm}$ GVO) in pump mode and compared with the simulation results, as shown in Figure 6. The test performance curve was expected to be stable with a negative slope when the flow rate was larger than $340 \mathrm{~kg} / \mathrm{s}$. However, a hump region marked by a positive slope was also found during part-load conditions. The numerical simulation head of the prototype shows a good agreement with the test curve (the maximum error is 6.4\%), which proves the numerical calculation is reliable and is the foundation of the following detailed studies. Therefore, the same numerical method was adopted for the splitter blade runner scheme and the head is also presented in Figure 6.

Because the efficiency obtained from ANSYS-CFX is only hydraulic efficiency without considering the electric motor and mechanical efficiency and volumetric efficiency, it cannot be compared with test system efficiency directly. Therefore, the difference in simulation hydraulic efficiency between the splitter blade runner scheme and the prototype is showed in Figure 7. It can be observed that the efficiency of splitter blade runner scheme is higher than that of the prototype at full flow rates, with the maximum difference of $2.4 \%$. Combining Figure 6 with Figure 7, it can be concluded that splitter blade runner scheme improves the head and efficiency of the pump-turbine in pump mode and reduces the hump region to a certain degree. As a result, four operation points $\mathrm{A}, \mathrm{B}, \mathrm{C}$, and D corresponding to flow rates $266 \mathrm{~kg} / \mathrm{s}, 311.3 \mathrm{~kg} / \mathrm{s}$, $391.4 \mathrm{~kg} / \mathrm{s}$, and $475.8 \mathrm{~kg} / \mathrm{s}$ were chosen to investigate the inner flow characteristics for a single guide vane opening in the following parts. 

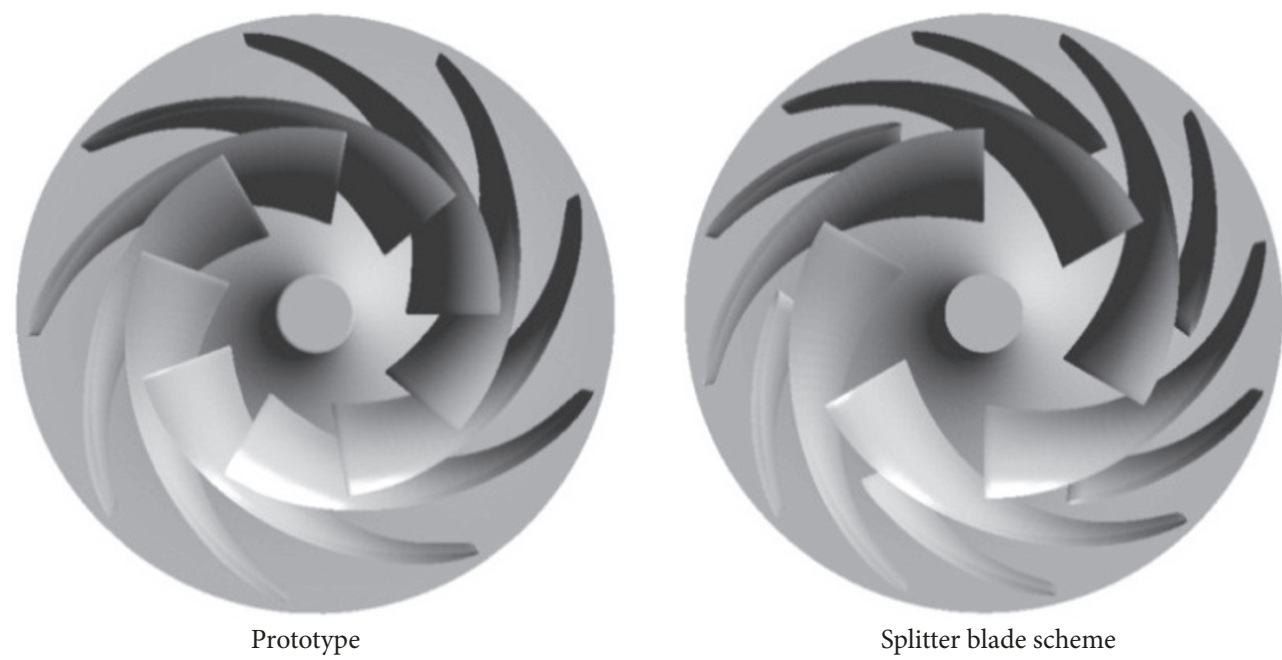

FIgURE 3: The prototype runner (left) and the splitter blade runner (right).
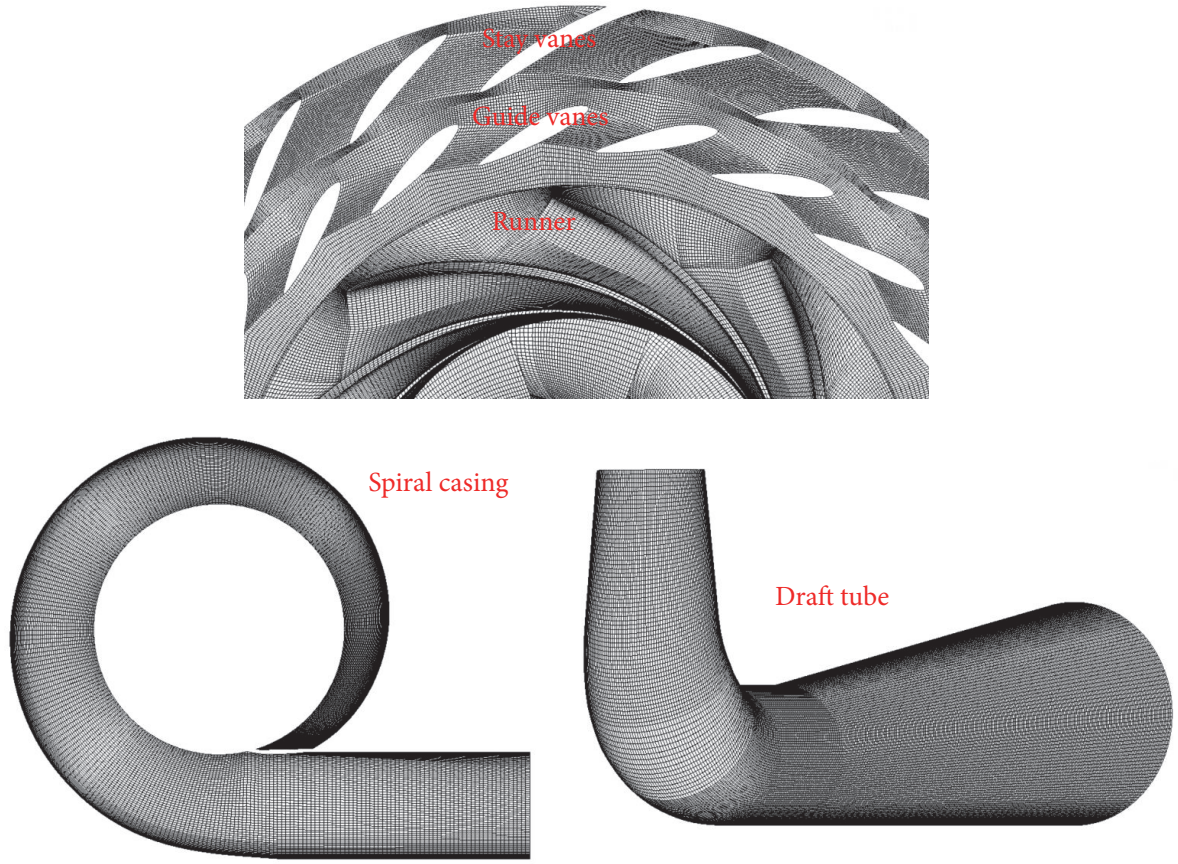

Figure 4: The mesh details of pump-turbine computation domain.

\section{Results and Discussions}

4.1. The Radial and Tangential Velocity Distributions at the Runner Outlet. The contours of the tangential component of absolute velocity at runner outlet, normalized by runner tip circumference velocity $U_{2}$, for the runners with and without splitter blade at four different flow rates are presented in Figure 8. According to Euler's equation, the theoretical head in pump mode equals $H_{t h}=\left(V_{t 2} U_{2}-V_{t 1} U_{1}\right) / g$, where $U_{1}$ and $U_{2}$ are the circumference velocity and $V_{\mathrm{t} 1}$ and $V_{\mathrm{t} 2}$ are the tangential velocity at runner inlet and outlet, respectively.
In this expression, $U_{2}$ is constant in two runner schemes and $V_{\mathrm{t} 1} U_{1}$ is very small compared with $V_{\mathrm{t} 2} U_{2}$. Therefore, the theoretical head is determined almost by the runner outlet tangential velocity $V_{\mathrm{t} 2}$. Generally, $V_{\mathrm{t} 2}$ decreases with increase of the flow rate for both runner schemes in Figure 8 . The distributions of local high velocity regions near shroud side and low velocity regions near hub side correspond to the passage positions at flow rates $391.4 \mathrm{~kg} / \mathrm{s}$ and $475.8 \mathrm{~kg} / \mathrm{s}$, whereas they exhibit more complex structure at $266 \mathrm{~kg} / \mathrm{s}$ and $311.3 \mathrm{~kg} / \mathrm{s}$. The tangential velocities in runner with splitter blade are evidently larger than that in runner without splitter 


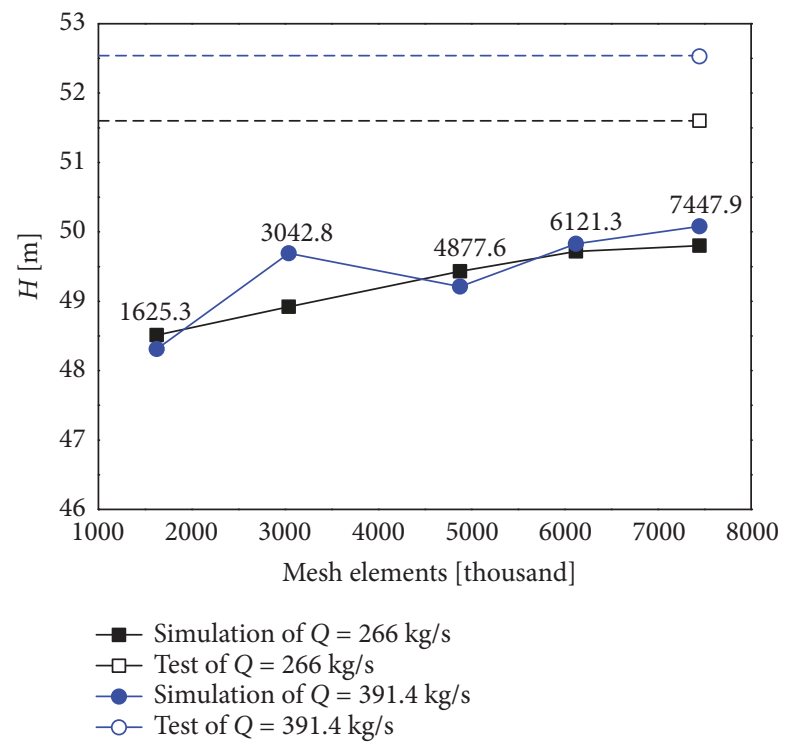

FIgURE 5: Mesh independence analysis at two flow rates.



FIGURE 6: The head curves comparison between tests and simulations.

blade, particularly at flow rates $266 \mathrm{~kg} / \mathrm{s}$ and $311.3 \mathrm{~kg} / \mathrm{s}$. Therefore, the splitter blade runner contributes to higher head in agreement with the performance curve in Figure 6.

Figure 9 presents the contours of the radial component of relative velocity at runner outlet at different flow rates, which is normalized by runner tip circumference velocity $U_{2}$. As can be seen, the distribution of radial velocity has an apparent relationship with the runner passages. The high and low radial velocity regions are distributed alternatively along the circumference direction of the runner outlet. This could be partly because the classical jet-wake pattern occurs at runner outlet region; that is, the high radial velocity appears near the pressure side of blade while the low radial velocity

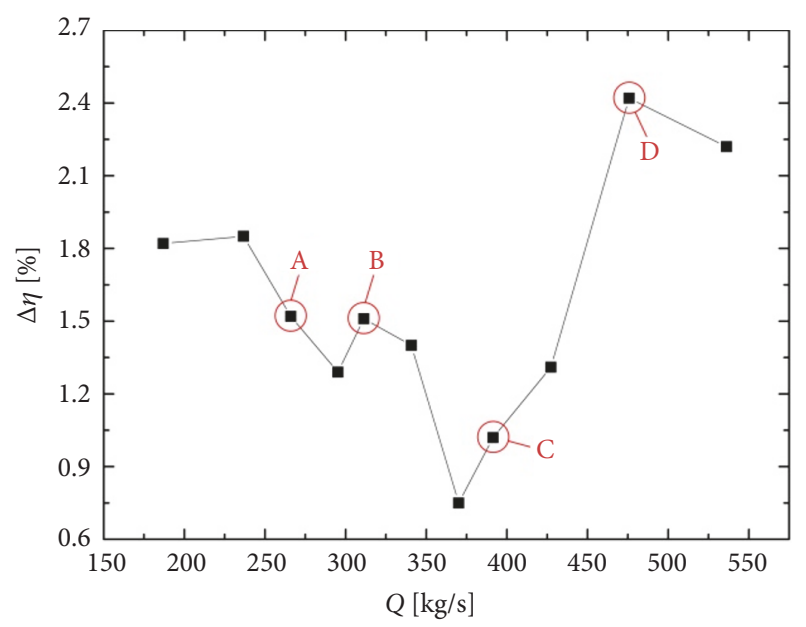

FIGURE 7: The simulation hydraulic efficiency difference between the splitter blade runner scheme and the prototype.

emerges adjacent to the suction side of blade, according to relevant references [23, 24]. For prototype runner at flow rate $475.8 \mathrm{~kg} / \mathrm{s}$, three high radial velocity regions located evenly at runner outlet can be observed from the passage midplane to the hub. When the flow rate reduces to $391.4 \mathrm{~kg} / \mathrm{s}$, the high radial velocity regions almost disappear whereas the area of low radial velocity increases even with negative values. That is, some small backflows appear in these passages near shroud side. Then these backflow regions with negative radial velocity progressively move to hub side at $266 \mathrm{~kg} / \mathrm{s}$ and $311.3 \mathrm{~kg} / \mathrm{s}$ and extend with the decrease of flow rate. The backflow regions are blade wake in which the momentum interchanges strongly and the hydraulic loss exists. As a result, the hydraulic loss in prototype runner increases with the decrease of flow rate. As for the runner with splitter blade, the evolution of radial velocity with flow rate is similar to that of prototype runner, but with evidently smaller backflow regions, particularly at the flow rates $266 \mathrm{~kg} / \mathrm{s}$ and $311.3 \mathrm{~kg} / \mathrm{s}$ at which the backflow regions with negative radial velocity nearly disappear. The reason could be that the backflow is suppressed due to the improved jet-wake pattern by the splitter blade. It can be concluded that the splitter blade runner scheme optimizes the structure of jetwake pattern, decreases the hydraulic loss, and improves the runner performance, especially at low flow rates.

4.2. The Pressure and Streamline Distributions. The pressure and streamline distributions on the pressure side of runner blade are presented in Figure 10 at four different flow rates. The area of low-pressure zone at the leading edge in the prototype is evidently larger than that in splitter blade runner scheme at flow rate $266 \mathrm{~kg} / \mathrm{s}$. This indicates that the blade does not fully deliver energy on the fluid in this region. The reason could be that the blade number at the runner inlet of splitter blade runner scheme is less than that of the prototype, which decreases the inlet blockage and flow velocity, resulting in an increase of shock angle and static pressure. Meanwhile, according to streamline distribution of the main blade, the 

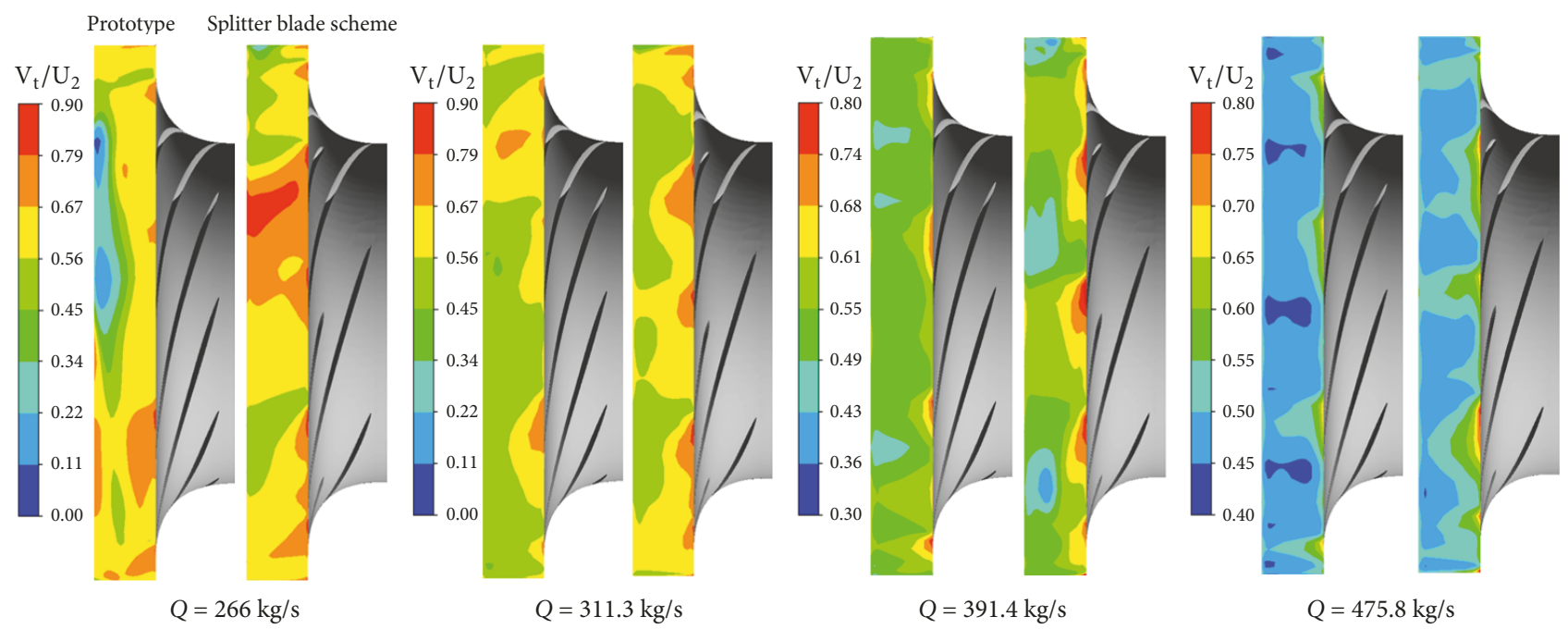

FIGURE 8: The contours of the tangential component of absolute velocity at runner outlet.
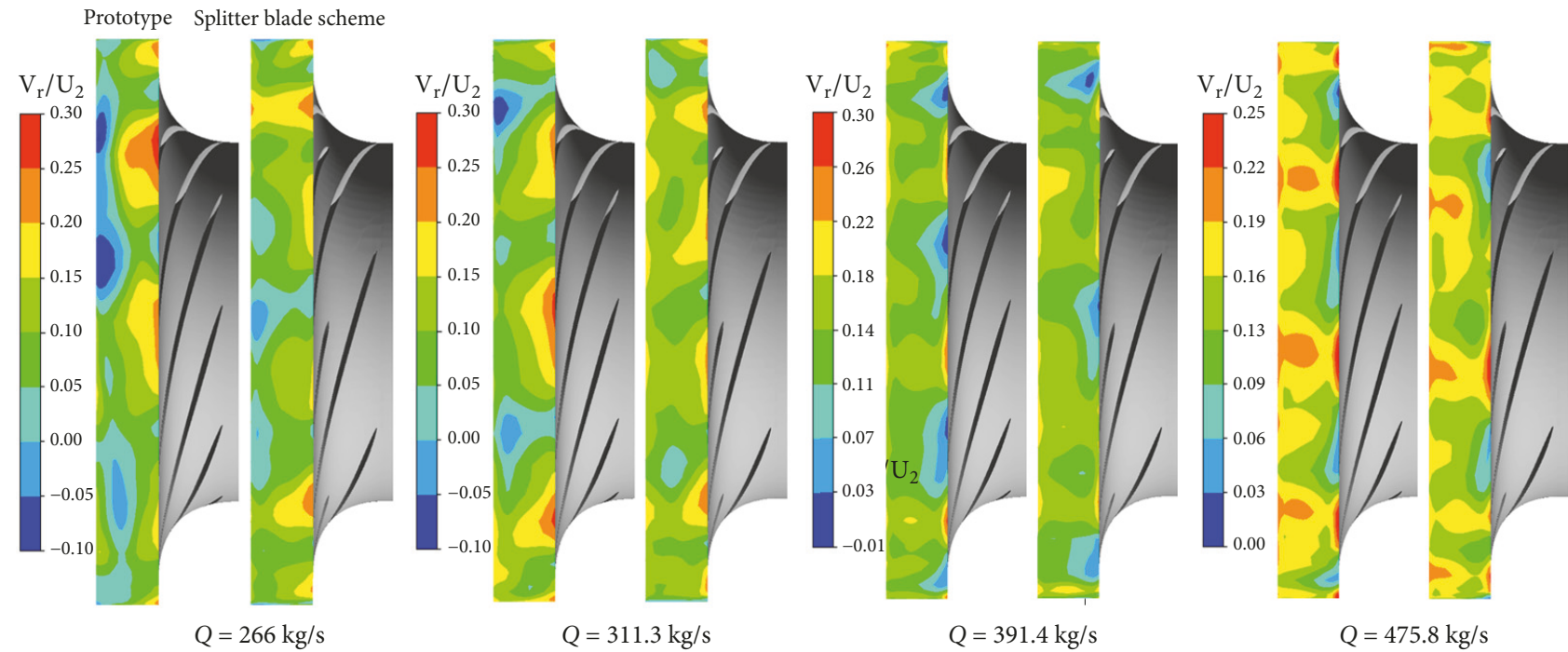

FIGURE 9: The contours of the radial component of relative velocity at runner outlet.

nonuniform streamline with a high curvature in prototype runner deviates from hub to shroud near the blade front part, which might be one of the reasons for the low-pressure region. Furthermore, the main blade of splitter blade runner scheme at the trailing edge has a larger high-pressure zone than the prototype, which contributes to a higher head.

In Figure 10, it can also be observed that the splitter blade runner scheme has better streamline distributions than the prototype on the pressure side of the main blade. The streamline distributions in both schemes are relatively smooth at larger flow rates $475.8 \mathrm{~kg} / \mathrm{s}$ and become distortion at the other three flow rates. Therefore, combining the unstable region of performance curve in Figure 6, detailed analyses are carried out at lower flow rate to reveal the effect of adding splitter blade on the internal flow characteristics and the performance in the following study.
4.3. The Entropy Generation Rate Distribution. The energy loss in a pump-turbine is caused by the effects of Reynolds stress and viscous stress. This process is irreversible and the lost energy converts to internal energy, resulting in the entropy generation. As recommended by Denton [25], entropy is an extremely useful method to measure the loss, which directly represents the devastation of useful work. Meanwhile, some relevant studies on the entropy generation rate $[25,26]$ were proposed to analyze unsteady turbulence flow situation in the operation process of fluid machinery. Moore [27] applied the entropy generation rate to the eddy viscosity turbulence flow model. In this model, the internal heating source was neglected and the entropy generation rate per volume was first defined in (1). The turbulent viscous dissipation term on the right hand in this equation was expressed by using eddy viscosity as in (2): 

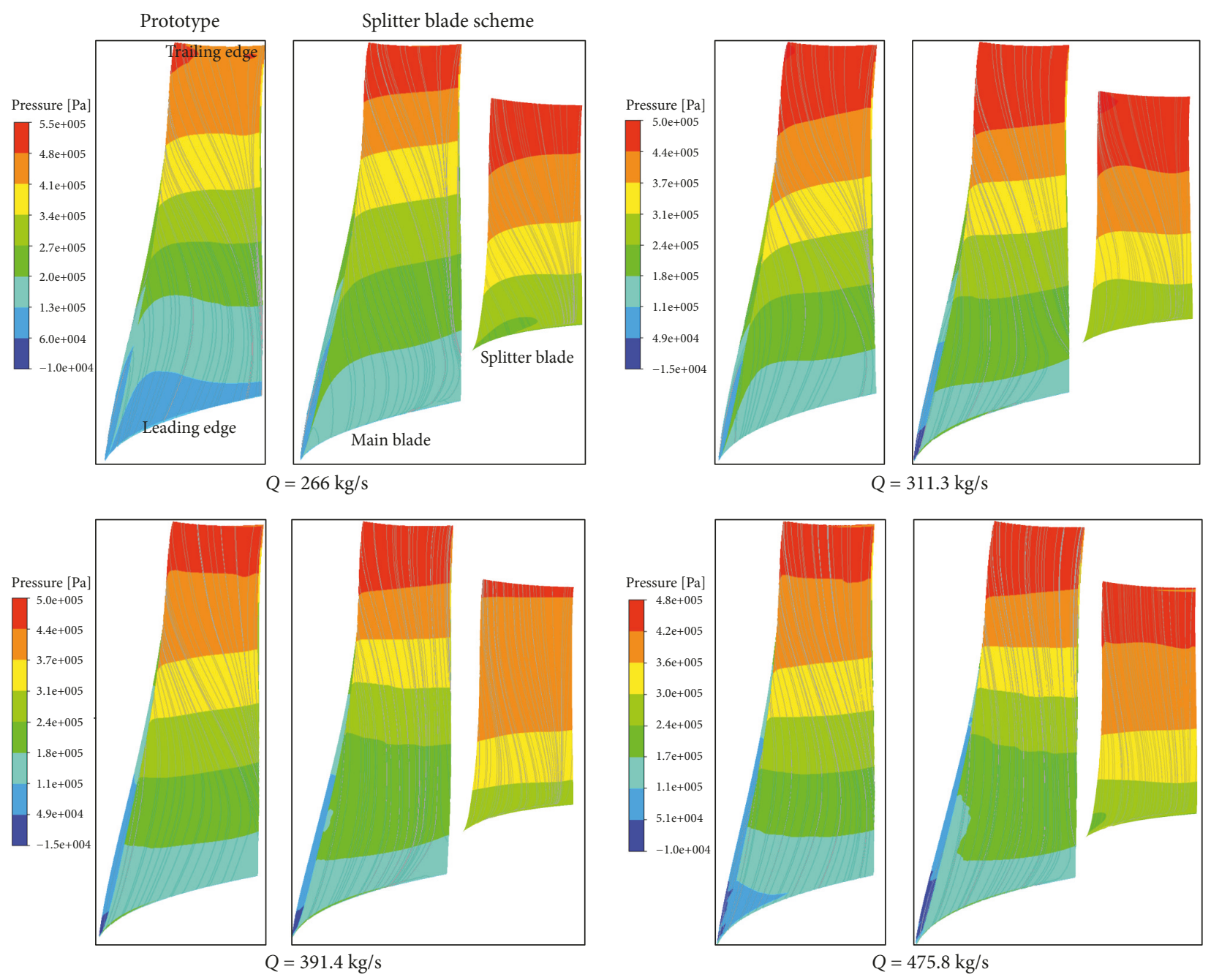

FIGURE 10: The contours of pressure and streamline distributions on the pressure side of runner blade.

$$
\begin{aligned}
\bar{T} \sigma & =\frac{k}{\bar{T}}\left[\left(\frac{\partial \bar{T}}{\partial x_{i}}\right)^{2}+\overline{\left(\frac{\partial T^{\prime}}{\partial x_{i}}\right)^{2}}\right]+\bar{\tau}_{i j} \frac{\partial \bar{u}_{i}}{\partial x_{j}}+\overline{\tau_{i j}^{\prime} \frac{\partial u_{i}^{\prime}}{\partial x_{j}}} \\
\overline{\tau_{i j}^{\prime} \frac{\partial u_{i}^{\prime}}{\partial x_{j}}} & =\frac{\mu_{t}}{\mu} \bar{\tau}_{i j} \frac{\partial \bar{u}_{i}}{\partial x_{j}}
\end{aligned}
$$

According to [28], the rotatory machinery model can be considered as adiabatic excluding the thermal diffusion effect. The final entropy generation rate per volume $\sigma$ is expressed as follows:

$$
\begin{aligned}
\sigma & =\frac{1}{T}\left(\bar{\tau}_{i j} \frac{\partial \bar{u}_{i}}{\partial x_{j}}+\frac{\mu_{t}}{\mu} \bar{\tau}_{i j} \frac{\partial \overline{u_{i}}}{\partial x_{j}}\right) \\
\tau_{i j} & =\mu\left(\frac{\partial u_{i}}{\partial x_{j}}+\frac{\partial u_{j}}{\partial x_{i}}-\frac{2}{3} \frac{\partial u_{k}}{\partial x_{k}} \delta_{i j}\right),
\end{aligned}
$$

where $\bar{\tau}_{i j}$ is the viscous stress sensor, $\mu_{t}$ and $\mu$ are eddy viscosity and turbulent dynamic viscosity, respectively, and
$T$ is temperature of working flow which is considered to be constant of $298 \mathrm{~K}$.

Figure 11 presents the distributions of entropy generation rate (EGR) per volume at mid span in runner, guide vanes, and stay vanes of the prototype and splitter blade runner scheme at flow rate $266 \mathrm{~kg} / \mathrm{s}$. Large entropy generation rate in the runner can be found at the leading edge of blade suction side due to small incident angle, in the passage, and at the trailing edge of main blades due to blade wake effect. The largest entropy generation rate concentrates on the interface between runner and guide vanes, the leading edge of guide vanes and the suction side of stay vanes, causing energy loss and a drop of efficiency. The area of high entropy generation rate in the splitter runner blade scheme is well smaller than that in prototype. This means that adding splitter blade can effectively reduce the energy loss in the runner, guide vanes, and stay vanes, conducing to the improvement of the efficiency.

4.4. The Vorticity Distribution at Guide Vanes and Stay Vanes. The vorticity can be used to indicate the local ration rate and 


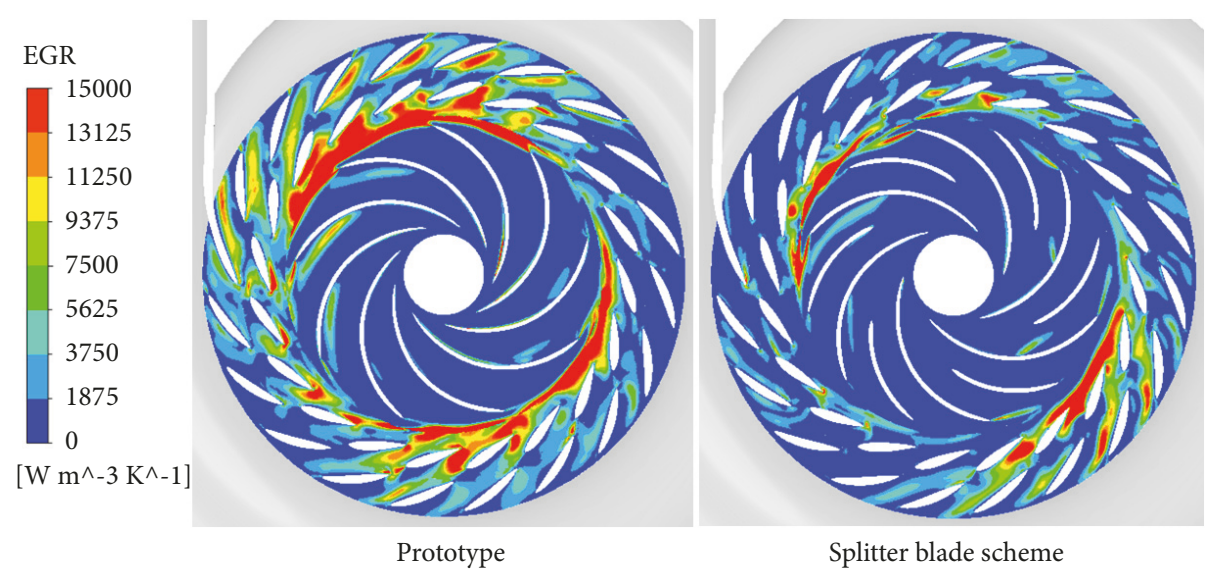

FIGURE 11: The contours of entropy generation rate distribution at mid span for both schemes.
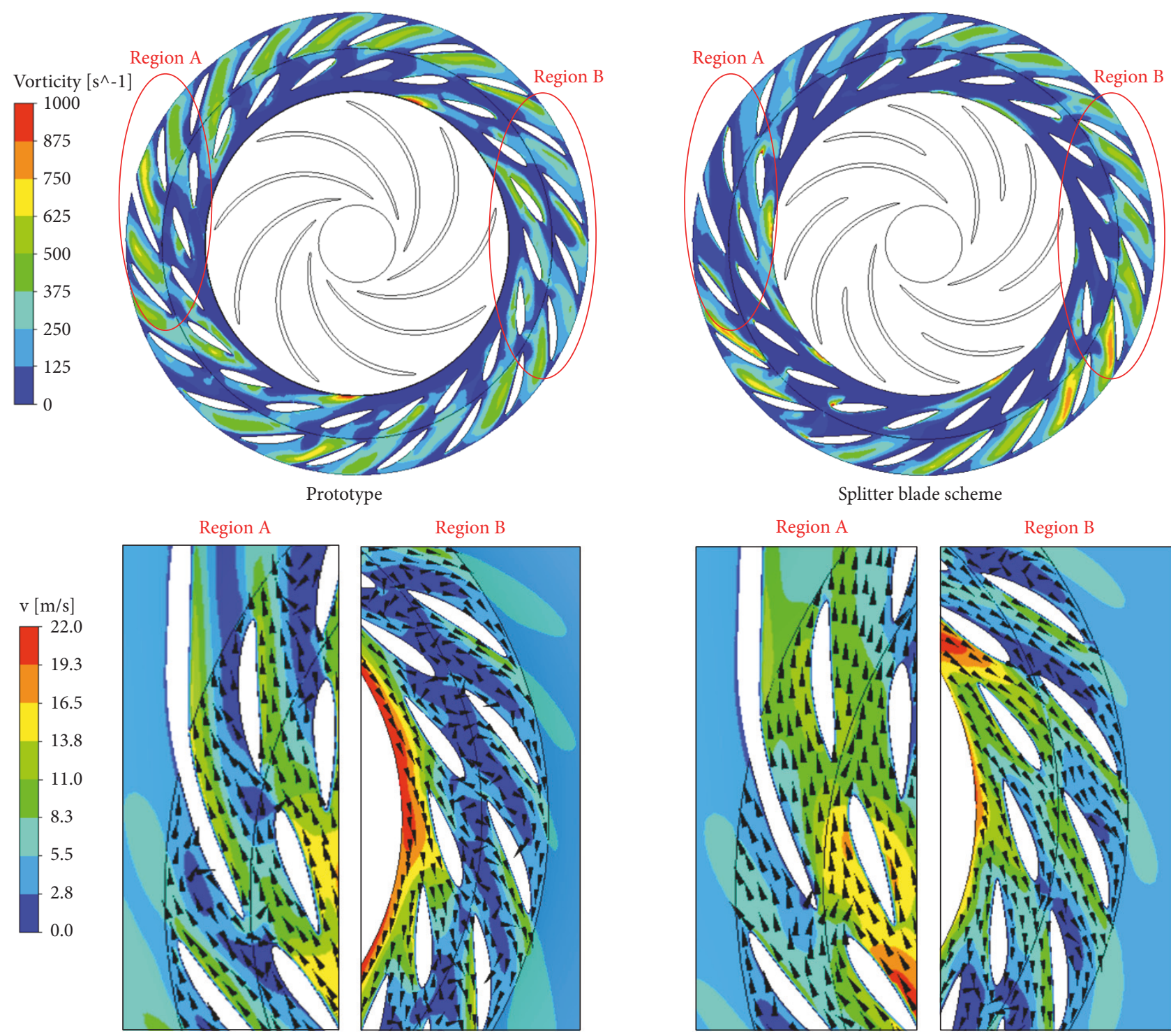

Prototype



Splitter blade scheme

FIGURE 12: The vorticity and velocity distributions of guide vanes and stay vanes at mid span. 
turbulence of the fluid. It can be defined by the following expression:

$$
\omega=\frac{\partial v_{y}}{\partial x}-\frac{\partial v_{x}}{\partial y} .
$$

Figure 12 shows the vorticity and velocity distributions of guide vanes and stay vanes at mid span in prototype and splitter blade runner scheme at flow rate $266 \mathrm{~kg} / \mathrm{s}$. As observed, the area of high vorticity in the prototype is well larger than that in splitter blade runner scheme, especially in region $\mathrm{A}$ and region $\mathrm{B}$, which is mainly due to the deviation effect of the incoming flow. The large vorticity indicates that fluid produces a large rotational angular velocity and intense perturbation in the flow field, which is not conducive to energy conversion and transportation. It may even be one of the significant reasons for the formation of the hump region in the prototype. Meanwhile, according to the local velocity distributions in regions $\mathrm{A}$ and $\mathrm{B}$ in Figure 12, some small vortices and flow separation that can be found in the prototype improved in splitter blade scheme. This is because the splitter blade added at the rear part of the runner channel decreases the velocity slip, enhances the control to the fluid, optimizes the jet-wake flow patterns, and makes the flow coming out from runner more uniform. Then the uniform incoming flow reduces the impingement on the guide vane inlet and makes the flow flatter in the guide vane channels, which can effectively improve flow instability and flow separation phenomenon. As a result, the splitter blade runner scheme has smoother flow patterns and less energy loss in the guide vanes and stay vanes, contributing to better performance.

\section{Conclusions}

The effects of adopting runner without and with splitter blade on the performance of the pump-turbine in pump mode were investigated by means of the numerical simulations. The numerical calculations showed a good agreement with the test results in performance prediction. Various quantities, including the velocity, pressure, streamline distribution, entropy generation rate, and vorticity distribution, were adopted to verify and analyze internal flow characteristics inside the pump-turbine for different runner schemes. Some conclusions are drawn as follows:

(1) The runner with splitter blade can effectively improve the performance for a pump-turbine in pump mode. Compared to prototype runner, the maximum increased values of the head and the efficiency are $2 \mathrm{~m}$ and $2.4 \%$ at full operating conditions in splitter blade runner scheme, respectively

(2) When the flow rate is less than $391.4 \mathrm{~kg} / \mathrm{s}$, in prototype runner, the radial component of relative velocity at the runner outlet reduces to zero and even negative values, and the streamlines on the pressure side of the blade cause an obvious distortion, which result in serious backflow and hydraulic loss relative to splitter blade runner scheme

(3) The tangential component of absolute velocity at runner outlet and the distribution of high-pressure region on the pressure side of the blade in splitter blade runner scheme is evidently larger than that in runner without splitter blade, particularly at flow rates $266 \mathrm{~kg} / \mathrm{s}$ and $311.3 \mathrm{~kg} / \mathrm{s}$, contributing to higher head

(4) At lower flow rate $266 \mathrm{~kg} / \mathrm{s}$, the areas of high entropy generation rate and high vorticity in guide vanes and stay vanes for the splitter blade runner scheme are well smaller than those for the prototype scheme, which decreases the energy loss and enhances the energy conversion and transportation

\section{Nomenclature}

$g\left[\mathrm{~m} / \mathrm{s}^{2}\right]: \quad$ Gravitational acceleration

$H[\mathrm{~m}]: \quad$ Head

$H_{\mathrm{d}}[\mathrm{m}]: \quad$ Nominal head

$H_{\text {th }}[\mathrm{m}]: \quad$ Theoretical head

$k[\mathrm{~W} / \mathrm{m} \mathrm{K}]: \quad$ Thermal conductivity

$n[\mathrm{r} / \mathrm{min}]: \quad$ Rotational speed

$Q\left[\mathrm{~m}^{3} / \mathrm{h}\right]: \quad$ Mass flow rate

$Q_{d}\left[\mathrm{~m}^{3} / \mathrm{h}\right]: \quad$ Nominal flow rate

$T[\mathrm{~K}]: \quad$ Temperature

$U_{1}[\mathrm{~m} / \mathrm{s}]: \quad$ Circumferential velocity at runner inlet

$U_{2}[\mathrm{~m} / \mathrm{s}]: \quad$ Circumferential velocity at runner outlet

$u_{\mathrm{i}}[\mathrm{m} / \mathrm{s}]: \quad$ Liquid velocity in the $\mathrm{x} / \mathrm{y} / \mathrm{z}$ direction

$v[\mathrm{~m} / \mathrm{s}]: \quad$ Relative velocity

$V_{\mathrm{r}}[\mathrm{m} / \mathrm{s}]: \quad$ Radial component of relative velocity

$V_{\mathrm{t} 1}[\mathrm{~m} / \mathrm{s}]: \quad$ Tangential velocity at runner inlet

$V_{\mathrm{t} 2}[\mathrm{~m} / \mathrm{s}]: \quad$ Tangential velocity at runner outlet

$x_{\mathrm{j}}: \quad \mathrm{x} / \mathrm{y} / \mathrm{z}$ direction

$\Delta \eta: \quad$ The difference of hydraulic efficiency

$\mu\left[\mathrm{kg} \cdot \mathrm{m}^{-1} \cdot \mathrm{s}^{-1}\right]$ : Dynamic viscosity

$\mu_{\mathrm{t}}\left[\mathrm{kg} \cdot \mathrm{m}^{-1} \cdot \mathrm{s}^{-1}\right]$ : Eddy viscosity

$\sigma\left[\mathrm{W} \cdot \mathrm{m}^{-3} \cdot \mathrm{K}^{-1}\right]$ : Entropy generation rate per unit volume

$\bar{\tau}_{i j}$ : $\quad$ Viscous stress sensor

$\omega\left[\mathrm{s}^{-1}\right]: \quad$ Vorticity.

\section{Data Availability}

The data used to support the findings of this study are included within the article.

\section{Conflicts of Interest}

The authors declared no potential conflicts of interest with respect to the research, authorship, and/or publication of this article.

\section{Acknowledgments}

The authors disclosed receipt of the following financial support for the research, authorship, and/or publication of this article: This work was supported by the National Natural Science Foundation of China (Grant No. 51409123), China Postdoctoral Science Foundation (2015M581734), a project funded by the Priority Academic Program Development of Jiangsu Higher Education Institutions (PAPD), and Innovation Project for Postgraduates of Jiangsu Province (Grant No. KYLX15_1064). 


\section{References}

[1] G. Ardizzon, G. Cavazzini, and G. Pavesi, "A new generation of small hydro and pumped-hydro power plants: Advances and future challenges," Renewable \& Sustainable Energy Reviews, vol. 31, pp. 746-761, 2014.

[2] Z. Zuo, S. Liu, Y. Sun, and Y. Wu, "Pressure fluctuations in the vaneless space of High-head pump-turbines - A review," Renewable \& Sustainable Energy Reviews, vol. 41, pp. 965-974, 2015.

[3] J. P. Deane, B. P. Ó Gallachóir, and E. J. McKeogh, “Technoeconomic review of existing and new pumped hydro energy storage plant," Renewable \& Sustainable Energy Reviews, vol. 14, no. 4, pp. 1293-1302, 2010.

[4] P. Punys, R. Baublys, E. Kasiulis, A. Vaisvila, B. Pelikan, and J. Steller, "Assessment of renewable electricity generation by pumped storage power plants in EU Member States," Renewable \& Sustainable Energy Reviews, vol. 26, pp. 190-200, 2013.

[5] Z. Ming, F. Junjie, X. Song, W. Zhijie, Z. Xiaoli, and W. Yuejin, "Development of China's pumped storage plant and related policy analysis," Energy Policy, vol. 61, pp. 104-113, 2013.

[6] T. Hino and A. Lejeune, "Pumped storage hydropower developments," Comprehensive Renewable Energy, vol. 6, pp. 405-434, 2012.

[7] J. Yan, J. Koutnik, U. Seidel, and B. Hubner, "Compressible Simulation of Rotor-Stator Interaction in Pump-Turbines," The International Journal of Fluid Machinery and Systems, vol. 3, no. 4, pp. 315-323, 2010

[8] G. Olimstad, T. Nielsen, and B. Børresen, "Dependency on runner geometry for reversible-pump turbine characteristics in turbine mode of operation," Journal of Fluids Engineering, vol. 134, no. 12, 2012.

[9] J. Yin, D. Wang, X. Wei, and L. Wang, "Hydraulic Improvement to Eliminate S-Shaped Curve in Pump Turbine," Journal of Fluids Engineering, vol. 135, no. 7, p. 071105, 2013.

[10] W. Yang and R. Xiao, "Multiobjective optimization design of a pump-turbine impeller based on an inverse design using a combination optimization strategy," Journal of Fluids Engineering, vol. 136, no. 1, 2014.

[11] U. Ješe, R. Fortes-Patella, and M. Dular, "Numerical study of pump-turbine instabilities under pumping mode off-design conditions," in Proceedings of the ASME/JSME/KSME 2015 Joint Fluids Engineering Conference, AJKFluids 2015, Republic of Korea, July 2015.

[12] X. Zhang, Y. Cheng, L. Xia, J. Yang, and Z. Qian, "Looping Dynamic Characteristics of a Pump-Turbine in the S-shaped Region During Runaway," Journal of Fluids Engineering, vol. 138, no. 9, p. 091102, 2016.

[13] G. Kergourlay, M. Younsi, F. Bakir, and R. Rey, "Influence of splitter blades on the flow field of a centrifugal pump: test-analysis comparison," International Journal of Rotating Machinery, vol. 2007, Article ID 85024, 2007.

[14] G. Cavazzini, G. Pavesi, A. Santolin, G. Ardizzon, and R. Lorenzi, "Using splitter blades to improve suction performance of centrifugal impeller pumps," Proceedings of the Institution of Mechanical Engineers, Part A: Journal of Power and Energy, vol. 229, no. 3, pp. 309-323, 2015.

[15] S. A. Moussavi, A. Hajilouy Benisi, and M. Durali, "Effect of splitter leading edge location on performance of an automotive turbocharger compressor," Energy, vol. 123, pp. 511-520, 2017.
[16] X. He and X. Zheng, "Mechanisms of sweep on the performance of transonic centrifugal compressor impellers," Applied Sciences (Switzerland), vol. 7, no. 10, 2017.

[17] R. Du X, Q. Wang, and E. Yasuyuki, "Application of splitter blades runner pump turbine in Qing Yuan Pump Storage Station," Hydropower \& Pumped Storage, vol. 2, pp. 39-44, 2016.

[18] K. Tezuka, "Development and application of new type runner with splitter blades to pumped storage power plants," in 8th Asian International Fluid Machinery Conference, 2005.

[19] L. Meng, S. P. Zhang, L. J. Zhou, and Z. W. Wang, "Study on the Pressure Pulsation inside Runner with Splitter Blades in Ultra-High Head Turbine," IOP Conference Series: Earth and Environmental Science, vol. 22, no. 3, p. 032012, 2014.

[20] Wei Li, Xiaofan Zhao, Weiqiang Li, Weidong Shi, Leilei Ji, and Ling Zhou, "Numerical Prediction and Performance Experiment in an Engine Cooling Water Pump with Different Blade Outlet Widths," Mathematical Problems in Engineering, vol. 2017, Article ID 8945712, 11 pages, 2017.

[21] L. Deyou, W. Hongjie, X. Gaoming, G. Ruzhi, W. Xianzhu, and L. Zhansheng, "Unsteady simulation and analysis for hump characteristics of a pump turbine model," Journal of Renewable Energy, vol. 77, pp. 32-42, 2015.

[22] C. Widmer, T. Staubli, and N. Ledergerber, "Unstable characteristics and rotating stall in turbine brake operation of pumpturbines," Journal of Fluids Engineering, vol. 133, no. 4, pp. 4110141101-9, 2011.

[23] D. Eckardt, "Detailed flow investigations within a high-speed centrifugal compressor impeller," Journal of Fluids Engineering, vol. 98, no. 3, pp. 390-399, 1976.

[24] J. Keller, E. Blanco, R. Barrio, and J. Parrondo, "PIV measurements of the unsteady flow structures in a volute centrifugal pump at a high flow rate," Experiments in Fluids, vol. 55, no. 10, 2014.

[25] J. D. Denton, "The 1993 IGTI scholar lecture: loss mechanisms in turbomachines," Journal of Turbomachinery, vol. 115, no. 4, pp. 621-656, 1993.

[26] D. Li, H. Wang, Y. Qin, L. Han, X. Wei, and D. Qin, "Entropy production analysis of hysteresis characteristic of a pumpturbine model," Energy Conversion and Management, vol. 149, pp. 175-191, 2017.

[27] J. Moore and J. G. Moore, "Entropy Production Rates From Viscous Flow Calculations: Part I - A Turbulent Boundary Layer Flow," in Proceedings of the ASME 1983 International Gas Turbine Conference and Exhibit, p. V001T01A032, Phoenix, Arizona, USA.

[28] P. Newton, C. Copeland, R. Martinez-Botas, and M. Seiler, "An audit of aerodynamic loss in a double entry turbine under full and partial admission," International Journal of Heat and Fluid Flow, vol. 33, no. 1, pp. 70-80, 2012. 


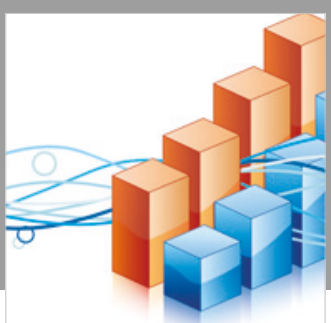

Advances in

Operations Research

\section{-n-m}
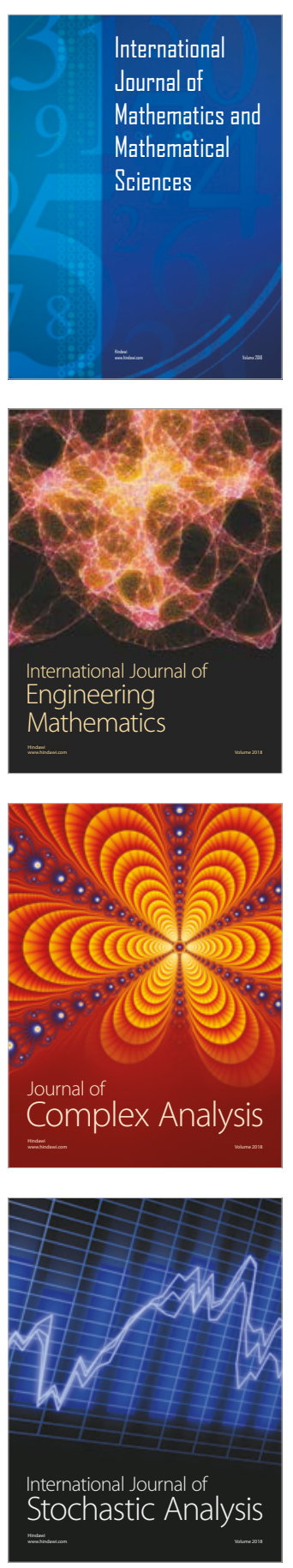


Journal of

Applied Mathematics
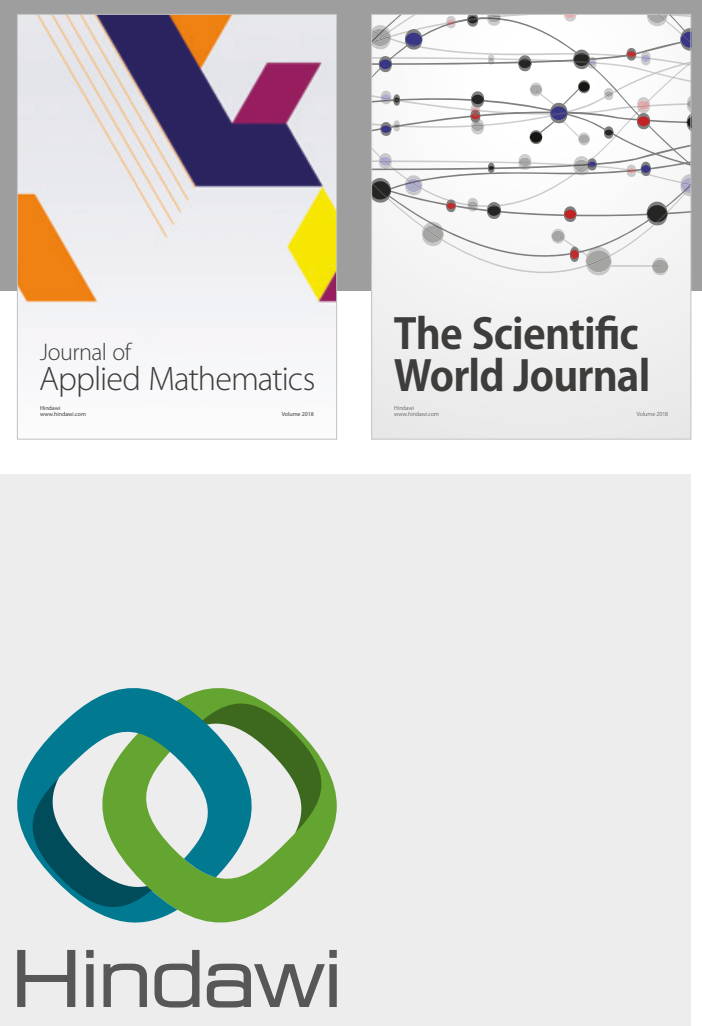

Submit your manuscripts at

www.hindawi.com

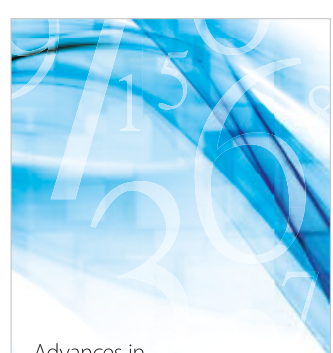

Advances in
Numerical Analysis


Mathematical Problems in Engineering



International Journal of

Differential Equations

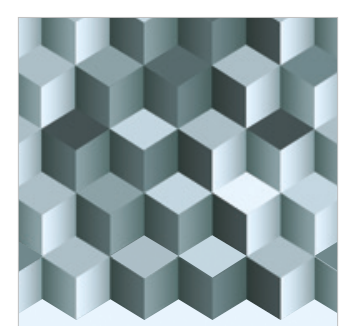

Journal of

Function Spaces



The Scientific

World Journal

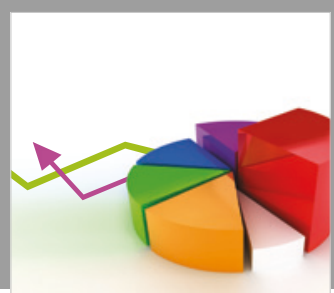

Journal of

Probability and Statistics
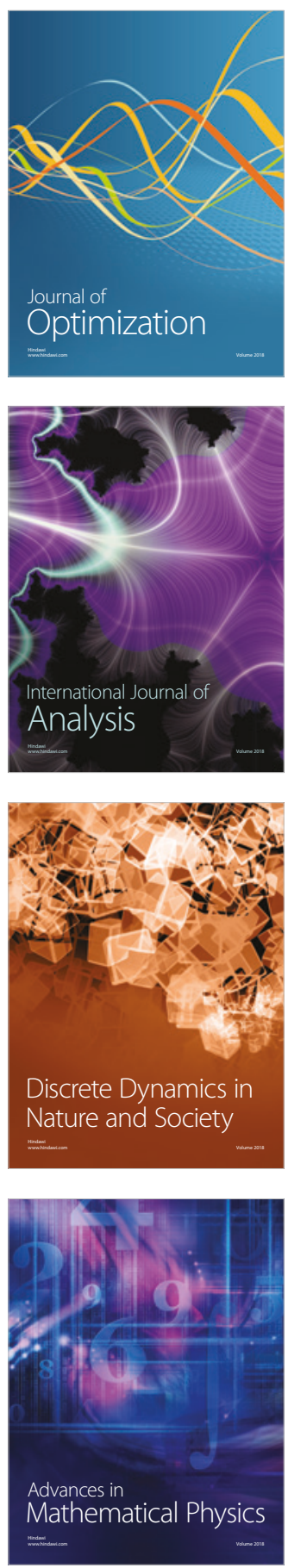